Review Paper http://ajol.info/index.php/ijbcs http://indexmedicus.afro.who.int

\title{
Contamination des céréales par l'aflatoxine en Afrique : revue des méthodes de lutte existantes
}

\author{
Eliasse DIEME ${ }^{1 *}$, Ramatoulaye FALL ${ }^{2}$, Ibrahima SARR ${ }^{3}$, Fallou SARR ${ }^{4}$, \\ Djibril TRAORE ${ }^{5}$ et Malang SEYDI ${ }^{6}$
}

\author{
${ }^{1}$ Institut de Technologie Alimentaire, Laboratoire de microbiologie alimentaire, BP 2765, Dakar, Sénégal. \\ ${ }^{2}$ Institut de Technologie Alimentaire, atelier céréales et légumineuses, BP 2765, Dakar, Sénégal. \\ ${ }^{3}$ Institut de Technologie Alimentaire, Laboratoire des mycotoxines, BP 2765, Dakar, Sénégal. \\ ${ }^{4}$ Institut Sénégalais de Recherche Agricole (ISRA), BP 3120 - Dakar. \\ ${ }^{5}$ Université Cheikh Anta Diop, Faculté des Sciences et Technique, Département de Biologie végétale, BP 5005, \\ Dakar, Sénégal. \\ ${ }^{6}$ EISMV de Dakar, BP 5077, Dakar, Sénégal. \\ *Auteur correspondant, E-mail :eliassedim@yahoo.fr ; Tél.: (221)77 71528 81;77010 5043.
}

\section{RESUME}

Les aflatoxines sont des toxines produites principalement par les moisissures du genre Aspergillus (Aspergillus flavus et Aspergillus parasiticus). Les molécules les plus abondantes de la famille des aflatoxines sont l'AFB1, l'AFB2, l'AFG1 et l'AFG2. C'est dans les pays africains, d'Asie du Sud et d'Amérique du Sud, où le climat est chaud et humide, que la contamination des céréales par l'aflatoxine est plus fréquente. Dans la plus part des pays africains, près de la moitié de la production céréalière a une teneur en aflatoxine supérieure aux standards internationaux. L'estimation de la quantité d'aflatoxine dans le maïs en 2016 a donné des intervalles de [1,06-852,2ppm] au Sénégal, de [8,0-1081ppm] en Tanzanie, avec un taux d'échantillons dépassant la limite maximale qui varie entre $2-85 \%$ de 622 échantillons. Au niveau africain la contamination des céréales par cette toxine a des conséquences économiques, alimentaires et surtout sanitaires. Pour lutter contre la présence de l'aflatoxine dans des céréales en Afrique des méthodes chimiques, physiques et biologiques sont employées, bien qu'elles soient limitées.

(c) 2016 International Formulae Group. All rights reserved.

Mot clés : Aflatoxine, Contamination, Céréale, Lutte, Prévalence, Afrique.

\section{African crops contamination by aflatoxin: review of existing methods of control}

\begin{abstract}
Aflatoxins are toxins produced mainly by Aspergillus (Aspergillus flavus and Aspergillus parasiticus). The most abundant molecules of aflatoxins family are AFB1, AFB2, AFG1 and AFG2. It is in African countries, South Asia and South America, where the climate is hot and humid, that contamination of crops with aflatoxin is more common. In most African countries, nearly half of crops production has higher aflatoxin content compared to international standards. The estimate of the quantity of aflatoxin in crops on the year 2016
\end{abstract}


has yielded intervals [1.06-852.2ppb] in Senegal, [8.0-1081ppb] in Tanzania, with a sample rate exceeding the maximum limit that varies between $2-85 \%$ of 622 samples. The level of grain aflatoxin contamination in Africa has economic and food consequences; and especially impact the human health. To fight against the contamination of Africa crops by aflatoxin, physical, chemical and biological methods are employed with obvious limits.

(C) 2016 International Formulae Group. All rights reserved.

Keywords : Aflatoxin, Contamination, crops, control, Prevalence, Africa.

\section{INTRODUCTION}

Les mycotoxines représentent des défis majeurs pour les systèmes mondiaux de sécurité alimentaire, la santé, la nutrition et les économies, car elles sont produites tant au cours de la production agricole, de la récolte, $\mathrm{du}$ transport, du stockage que de la transformation des aliments (Murphy et al., 2006). La contamination par l'aflatoxine des principaux aliments de consommation courante, à savoir le maïs, les arachides et le sorgho, atteint des niveaux inacceptables pour la santé dans de nombreux pays d'Afrique. La contamination par l'aflatoxine des aliments de consommation courante peut affecter la production du secteur agricole, en général, et chacun des quatre piliers de la sécurité alimentaire (disponibilité, accès, qualité de l'alimentation et régularité), en particulier.

En Afrique ces substances toxiques pour l'homme posent un réel problème de santé publique. Les populations Africaines sont exposées à l'aflatoxine même avant leur naissance (Williams et al., 2004; Wild and Gong, 2010). En général, les informations sur la prévalence du risque de contamination par l'aflatoxine en Afrique sont limitées. Mais les données disponibles indiquent que les niveaux d'aflatoxine dépassent souvent les limites de tolérance établies pour les aliments de base tels que les céréales (maïs, sorgho). Deux contraintes majeures plaident pour cette concentration élevée d'aflatoxine dans les céréales en Afrique: les conditions climatiques en Afrique sont favorables à la prolifération des Aspergillus et à la production d'aflatoxine. Cette contrainte naturelle est liée à la position géographique de l'Afrique qui est située entre les latitudes $40^{\circ} \mathrm{N}$ et $40^{\circ} \mathrm{S}$. Aussi la pauvreté et l'insécurité alimentaire font que pratiquement toutes les pratiques et politiques agricoles en Afrique sont orientées vers l'augmentation de la production céréalière sans pour autant se soucier de la qualité de cette dernière. Ainsi l'aflatoxine est un fléau qui gangrène la production céréalière en Afrique (Shephard, 2003; Bankole et Adebanjo, 2003; Bankole et al., 2006; Wagacha et Muthomi, 2008).

Les connaissances avérées des effets néfastes de l'aflatoxine sur la santé, justifiées d'une part par des événements sanitaires liés à la consommation de céréales contaminées par l'aflatoxine en Afrique (contamination 317 personnes par l'aflatoxine après consommation de maïs 125 d'entre eux meurent après hospitalisation au Kenya (CDC, 2004 ; Lewis et al., 2005), ont accentué la lutte institutionnelle et scientifique contre l'aflatoxine dans les céréales dans ce continent. Malgré cette volonté politique et scientifique, la présence d'aflatoxine avec parfois de fortes doses, est notée dans la production céréalière africaine au point qu'on s'interroge sur l'efficacité des méthodes de lutte employées.

Le travail que nous vous proposons consiste à faire une revue générale de toutes les méthodes de lutte déployées en Afrique pour réduire la concentration d'aflatoxine dans les céréales tout en montrant leur limite.

\section{DESCRIPTION ET ORIGINE DES AFLATOXINES}

Les aflatoxines sont des toxines naturelles produites par certaines souches du genre Aspergillus (CODEX, 2013). Cette famille de mycotoxines a été découverte lors 
d'investigations menées au moment de l'épisode de la "maladie X du dindon" (the Turkey X disease) qui a sévi en 1960 en Angleterre. L'analyse des matières premières constituant la nourriture de ces volailles avait révélé une contamination par Aspergillus flavus et mis en évidence la présence d'une toxine dans les tourteaux d'arachides : l'aflatoxine.

Les aflatoxines sont principalement produites par des espèces d'Aspergillus telles que Aspergillus flavus et Aspergillus parasiticus. Ces deux espèces sont toutes deux productrices d'aflatoxines de type B mais l'espèce Aspergillus parasiticus produit également des aflatoxines de type G (Varga et al., 2011). La famille des aflatoxines comprend plusieurs molécules. Les plus abondantes dans la nature sont les aflatoxines AFB1, AFB2, AFG1 et AFG2 (Huffman et al., 2010). Les aflatoxines M1 et M2 sont les métabolites des aflatoxines B1 et B2, issus de leur hydroxylation dans le foie des animaux exposés.

Parmi toutes les mycotoxines, l'aflatoxine B1 (AFB1) produite par Aspergillus flavus et Aspergillus parasiticus est la forme la plus fréquente et la plus toxique (hépatotoxique, tératogène et mutagène) pour les mammifères (Tabuc, 2007). Le pouvoir toxique des aflatoxines est principalement associé à l'aflatoxine B1 qui est considérée comme le principal métabolite génotoxique et qui possède le plus fort potentiel cancérogène de toutes les aflatoxines. En 1993, le CIRC a classé l'aflatoxine B1 dans le groupe 1 (agent cancérogène), l'aflatoxine M1 dans le groupe 2B (agent peut-être cancérogène) et l'aflatoxine G1 dans le groupe 3 (agent inclassable quant à sa cancérogénicité) (IARC, 1997). La présence d'une double liaison sur le cycle dihydrofurane de l'AFB1 et de l'AFG1 serait à l'origine de la différence de toxicité avec leurs homologues AFB2 et AFG2. Par contre la différence de toxicité entre les aflatoxines du groupe $B$ et $G$ proviendrait de la substitution du cycle cyclopentone par un cycle lactone (Lee et al., 1981). Ces aflatoxines sont structurellement proches constitués par un assemblage d'une coumarine et de 3 furanes.

\section{LA CONTAMINATION DES CEREALES PAR L'AFLATOXINE EN AFRIQUE}

Ce sont dans les pays aux conditions climatiques chaudes et humides (Pays africains, Asie du Sud et Amérique du Sud) que la croissance des aspergillus est la plus favorisée. Ainsi, le riz, le maïs et le millet, aliments de base des populations de ces pays, sont souvent contaminés par les Aflatoxines. Aspergillus flavus est la moisissure qui est fréquemment incriminée lorsqu'il y'a contamination des céréales par l'aflatoxine. Aspergillus n'est que rarement capable d'infecter une plante saine ou le tissu des fruits à coque. L'infection par Aspergillus et la production d'aflatoxines qui en résulte dépendent du stress subi par la plante et des dommages causés par les insectes et les ravageurs. L'humidité ambiante et les températures optimales favorisent la prolifération des champignons. La prolifération fongique et la production d'aflatoxine ont lieu au champ et au cours du stockage. Au champ, les insectes attaquent la surface des grains facilitant l'accès des moisissures aux structures internes des céréales qui contiennent les nutriments et représentent ainsi un facteur important de risque (Afssa, 2009).

Parmi les céréales, le maïs est plus vulnérable à une contamination par Aspergillus que le mil ou le sorgho. En Afrique occidentale, par exemple, les concentrations en aflatoxines dans le millet perlé et le sorgho sont substantiellement plus faibles que dans le maïs (Kane et al., 1991 ; Bandyopadhyay et al., 2007). Le maïs est très fréquemment contaminé par des espèces d'aspergillus aflatoxinogénes (Aspergillus fluvus) en Afrique (Odhiambo et al., 2013).

Aussi La sensibilité du sorgho bicolore aux champignons à travers le monde est bien établie. Dans la plus part des cas, la 
contamination du sorgho par les moisissures aflatoxinogènes a lieu lors de l'entreposage de cette céréale (Javis, 1971 ; Elegbede, 1978). Aspergillus est parmi les champignons les plus fréquemment identifiés au niveau des grains en entreposage en Afrique (Abu Agla, 2002, Ahmed et al., 2005, Ahmed et al., 2008, Ahmed et al., 2009 et Makun et al., 2009). L'aflatoxine est parmi les mycotoxines les plus courantes identifiées dans le sorgho (Ryle, 2010).

La présence de Aspergillus flavus au niveau des céréales se manifeste par une couleur vert-olive ou vert-gris sur les grains. Cependant il n'est pas possible de détecter à l'œil nu des céréales infectées par les aflatoxines. Toutes les céréales moisies ne sont pas infectées par les aflatoxines, mais le risque de contamination par les aflatoxines est plus élevé sur les céréales gâtées et moisies que sur des grains présentant peu de moisissure.

Parmi les facteurs qui exacerbent la contamination des céréales par les Aspergillus puis par l'aflatoxine en Afrique on peut noter les récoltes à surmaturité, le séchage retardé et les grains endommagés pendant l'égrainage. Aussi l'entreposage des grains récoltés dont la teneur en humidité est $>10 \%$ et pendant des périodes prolongées dans des installations inadéquates entraînent la prolifération des moisissures dans les grains (Ahmed et al., 2009). De même, la pratique inappropriée consistant à mélanger des grains de grades différents pour améliorer la qualité des grains contaminés, quand certains d'entre eux contiennent un grand nombre de spores fongiques sera source d'inoculum pour le grain de bonne qualité et contaminera probablement les grains exemptés de toxines (Wagacha et Muthomi, 2008). Selon Alakonya et al. (2009), il est essentiel de réduire la contamination des cultures au champ, car le développement des champignons et la production d'aflatoxines se poursuivent à un rythme encore plus rapide lors des étapes post-récolte et de stockage. En résumé, les mauvaises pratiques agricole en
Afrique sont à l'origine de la contamination des céréales par l'aflatoxine (Akowuah et al., 2015). A ces facteurs viennent s'ajouter la pauvreté, l'ignorance de l'existence de l'aflatoxine dans les céréales de certains peuples et le faible niveau d'application de la règlementation sur l'aflatoxine en Afrique.

\section{L'AMPLEUR DE LA CONTAMINATION}

En Afrique orientale, l'exposition à l'aflatoxine est directement corrélée à la consommation journalière de maïs (Kimanya et al., 2008). Depuis 2009, des études relatives à la contamination par l'aflatoxine du maïs ont été menées dans différentes régions du Kenya. Les résultats, qui ont mis en évidence des niveaux alarmants de contamination du maïs par l'aflatoxine, ont fait l'objet de discussions avec des agriculteurs et autres acteurs de la filière, dont la Fédération nationale des producteurs agricoles du Kenya (Kenya National Federation of Agricultural Producers), qui se sont tous accordés à reconnaitre que la lutte contre la contamination du maïs par l'aflatoxine devait être une priorité.

Les niveaux alarmant de contamination du maïs par l'aflatoxine sont aussi notés dans d'autres pays en Afrique. En effet l'estimation de la quantité d'aflatoxine dans le maïs, révélé par AfricaAIMS et C-SAAP, 2016, a donnée des intervalles de [1,06-852,2ppb] au Sénégal, de [8,0-1081ppb] en Tanzanie, avec un taux d'échantillons dépassant la limite maximale qui varie entre $2-85 \%$ de 622 échantillons. Selon la même source, en Ouganda la quantité d'aflatoxine dans le maïs est de [86-3300ppb avec 20-65\% d'échantillons dépassant la limite maximale sur 100. Celle trouvée dans le sorgho varie entre $25 \mathrm{ppb}$ et $514 \mathrm{ppb}$ avec 65 $100 \%$ d'échantillons dépassant la limite maximale. La contamination des céréales (maïs, sorgho) par l'aflatoxine est donc répandue en Afrique et a été étudié dans plusieurs pays. Dans la plus part de ces pays, près de la moitié de la production céréalière a une teneur en aflatoxine supérieure aux standards internationaux. Par exemple au 
Kenya sur un total de 342 échantillons de maïs, $182(53,2 \%)$ avaient un plus grand niveau que celui du Département de l'Agriculture Américain (USDA) et celui de l'OMS dont le niveau acceptable est 20 parties par milliard (ppb) d'aflatoxine (CDC, 2004). Les aflatoxines ayant des concentrations jusqu'au $1.020 \mathrm{ppb}$ ont été signalées dans les grains au Malawi (Moss, 2008). Les valeurs moyennes de la contamination des céréales par l'aflatoxine B1 (FB1) au Nigéria sont $257.82 \mathrm{ppm}$ pour le maïs, $2587.47 \pm 78.23 \mathrm{ppm}$ pour le millet et $82.5 \pm 16.9 \mathrm{ppm}$ pour le riz avec une incidence très forte dans le cas du millet et du maïs (Atehnkeng et al., 2008; Makun et al., 2007; Makun et al., 2011). L'analyse des différents résultats ci- dessus montre que la contamination des céréales par l'aflatoxine varie suivant les localités mais aussi suivant les saisons. Dans tous les cas elle est généralement très élevée partout en Afrique. Cette présence d'aflatoxine à des concentrations élevées dans les céréales est le principal caractère qui ternit la qualité de la production céréalière dans le continent.

\section{LES CONSEQUENCES DE LA CONTAMINATION}

Par rapport à la réglementation internationale sur l'aflatoxine, la contamination des céréales par cette toxine a des conséquences économiques, alimentaires et sanitaires. En Afrique, l'impact sanitaire est prépondérant puisque la culture céréalière étant l'apanage de petits agriculteurs (cultures familiales), la production est généralement destinée à l'autoconsommation. Les céréales (maïs, mil et sorgho) sont à la base du système alimentaire de la quasi-totalité des pays d'Afrique.

En Afrique de l'Ouest, le maïs représente la principale source d'apport calorique dans le régime alimentaire national de presque tous les pays de la Zone (FAOSTAT, 2015). Cette forte dépendance alimentaire des populations de ces pays vis-àvis du maïs les expose à une contamination certaine par l'aflatoxine. Une étude menée par
Watson et al. (2015) au Sénégal sur la teneur en aflatoxine dans le sang des personnes suivant la fréquence de consommation du maïs a donné les moyennes géométriques suivantes : $41,1 \mathrm{pg} / \mathrm{mg}$ pour une fréquence de consommation inférieure à 4 jours par semaine, $61,4 \mathrm{pg} / \mathrm{mg}$ pour une fréquence de consommation supérieure ou égale à 4 jours par semaine. A travers cette étude, ils ont conclu que plus la fréquence de consommation de maïs augmente plus la quantité d'aflatoxine dans le sang est importante. La fréquence de consommation des céréales est très élevée en Afrique de l'Ouest. De ce fait même si dans certains cas la Gambie par exemple (Shephard, 2008) la quantité d'aflatoxine dans les céréales (maïs et riz) est faible, celle-ci induit une forte accumulation d'aflatoxine dans le sang des populations.

En Afrique orientale, l'exposition à l'aflatoxine est directement corrélée à la consommation journalière de maïs (Kimanya et al., 2008). Une étude menée par Gong et al. (2003) au Bénin et au Togo a révélé la présence d'aflatoxine dans le sang des enfants de 1 à 3 ans dont l'alimentation est à base de céréales locales. Il en est de même pour des enfants de 4 à 6 mois au Kenya (Obade, 2015). L'exposition à l'aflatoxine de ces populations va induire des problèmes récurrents de santé liés aux aflatoxicoses. En effet suivant la dose d'aflatoxine ingérée on note deux types d'aflatoxicoses: une aflatoxicose aiguë ou une aflatoxicose chronique. L'aflatoxicose aiguë est associée à des incidences sporadiques de la consommation d'aliments fortement contaminé par l'aflatoxine. Le Kenya a connu plusieurs épisodes récurrents d'aflatoxicose aiguë chez les êtres humains et a enregistré des centaines de morts dans les quatre dernières décennies suite à une consommation de maïs contaminé par l'aflatoxine (1981, 2001, 2004 et 2005) (DN, 2001; CDC, 2004; Lewis et al., 2005). Le dosage d'aflatoxine dans le maïs à l'origine de ces aflatoxicoses a révélé des concentrations supérieurs à 20 
$\mu \mathrm{g} / \mathrm{kg} \quad$ (Azziz-Baumgartner et al., 2005; Muture et Ogana, 2005; Probst et al., 2007; Muthama et al., 2009). Le maïs de la région affectée contenait jusqu'à 4.400 ppb d'aflatoxine B1, ce qui est 440 fois plus élevé que le niveau de tolérance 10 ppb fixé par le Bureau de Standards du Kenya (Muthoni, 2009).

L'aflatoxicose chronique est liée à une ingestion modérée et répétée d'aflatoxine dans le temps. L'exposition chronique à des quantités faibles d'aflatoxine (l'aflatoxine B1 en particulier), est associée à un risque accru de développer le carcinome hépatocellulaire, ou cancer du foie, ainsi que les troubles de la fonction immunitaire, la malnutrition et une croissance ralentie chez les enfants. Selon Liu et $\mathrm{Wu}$ (2010), l'aflatoxine B1 est responsable de 4,6-28,2\% des carcinomes hépatocellulaire du monde. Il est estimé que plus de 5 milliards de personnes dans les pays en voie de développement dans le monde entier sont à risque d'exposition à l'aflatoxicose chronique (Strosnider et al., 2006; Shephard, 2008). Une grande partie des personnes exposées sont en Afrique (Figure 1).

L'exposition chronique à l'aflatoxine est aussi liée à des troubles de la fonction immunitaire, à la malnutrition et à une croissance ralentie chez les enfants. L'étude la plus préoccupante en Afrique de l'Ouest est celle qui a montré une corrélation importante entre l'exposition à l'aflatoxine dans les aliments (farine à base de céréales locales) et la croissance ralentie des enfants qui sont exposés à la toxine dès la période néo-natale (Gong et al., 2002). Un niveau élevé d'exposition à l'aflatoxine au moment du sevrage a affecté la croissance des enfants dans la République du Bénin et au Togo (Gong et al., 2003 ; Gong et al., 2004). Comme dans le cas du cancer du foie, la prévalence du retard de croissance des enfants est très élevée dans les régions du Sud-est d'Asie et de l'Afrique subsaharienne, où l'exposition aux aflatoxines par la consommation de la nourriture contaminée est répandue. Le Tableau 1 décrit une corrélation entre la teneur en aflatoxine du sang et la prévalence du retard de croissance des enfants dans quelques pays d'Afrique.

Le double fardeau de l'exposition chronique aux mycotoxines et de l'insuffisance alimentaire augmente la mortalité et la morbidité, spécialement chez les enfants (Bryden, 2007 ; IARC, 2012).

Les agriculteurs et autres acteurs ont également subi de lourdes pertes financières. Par exemple, en $2010 ; 2,3$ millions de sacs de maïs de $90 \mathrm{~kg}$ ont été déclarés impropres à l'alimentation humaine et animale en raison de leur contamination par l'aflatoxine. L'institut international de recherche sur les cultures des zones tropicales semi-arides ICRISAT (2002) a communiqué le volume suivant des pertes de grains alimentaires dues aux mycotoxines surtout l'aflatoxine chaque année dans le monde: maïs 16 millions de tonnes, riz 12 millions de tonnes, arachides 1,8 millions de tonnes, sorgho et millet 378,000 tonnes, coprah 3,7 millions de tonnes, soja 2,3 millions de tonnes. Une part importante de ces pertes se produit dans les pays en voie de développement d'Asie et d'Afrique.

\section{LES MECANISMES DE LUTTE CONTRE LA CONTAMINATION DES CEREALES PAR L'AFLATOXINE EN AFRIQUE}

\section{Les mécanismes institutionnels}

Pour protéger les consommateurs contre les risques liés aux mycotoxines, un grand nombre de pays dont 15 pays africains ont légiféré sur certaines mycotoxines notamment les aflatoxines (Fellinger, 2006; Njobeh et al., 2010). D'après ces auteurs, les limites tolérables maximales pour les aflatoxines dans les aliments de consommation humaine en Afrique varient de 5 à $20 \mathrm{ppb}$ alors que pour les aliments de consommation animale, elles varient de 5 à $300 \mathrm{ppb}$. La réglementation est plus sévère pour les aliments pour nourrissons (0-10 ppb). L'élaboration de ces législations pour lutter contre l'aflatoxine dans un aliment révèle la 
volonté des pouvoirs publics d'un pays de protéger la population contre cette toxine. Cette volonté est souvent stimulée par l'ampleur du niveau de contamination en aflatoxine d'un aliment de consommation de base comme le cas des céréales en Afrique.

$\mathrm{Au}$ Ghana par exemple, les normes suivantes existent pour l'aflatoxine : maïs 15 $\mu \mathrm{g} / \mathrm{kg}$ (GS 211: 2013); semoule de maïs dégermée $20 \mu \mathrm{g} / \mathrm{kg}$ (GS 729: 2003). Le Ghana est l'un des rares pays de la CEDEAO à avoir traduit certaines normes en procédures opérationnelles normalisées. Le Nigéria dispose aussi depuis 2014 d'une politique nationale sur la sécurité alimentaire. Les teneurs maximales en aflatoxine telle qu'elles sont fixées par l'organisation de normalisation du Nigéria sont : semoule de maïs (norme NIS 718 : 2010) teneur max en AFB1 est 2ppb, grains de sorgho (norme NIS 328: 2003) teneur max 10ppb, grains de mil (norme NIS 467 : 2003) teneur max 10ppb et pour les grains de maïs la norme NIS 253 : 2003 est en cour de révision; $4 \mathrm{ppb}$ pour l'aflatoxine totale et $2 \mathrm{ppb}$ pour l'aflatoxine B1. Cette même teneur maximale en aflatoxine totale et en AFB1 dans les céréales a été aussi instaurée au Bénin par la loi 09-1984. Tous les autres pays de la zone CEDEAO utilisent les limites du codex alimentaire ou celles instaurées par des partenaires commerciaux. L'application des ces mesures (normes nationaux ou limites $\mathrm{du}$ codex) visant à réduire la teneur des aliments (céréales) en aflatoxine dans ces différents pays, est l'œuvre des autorités institutionnelles. Ces autorités institutionnelles disposent dans la plus part de ces pays des organismes et laboratoires impliqués dans la lutte contre l'aflatoxine (ECOWAS, 2015).

\section{LES METHODES SCIENTIFIQUES Les méthodes chimiques}

Les produits toxiques et « d'arôme anormal » utilisés dans les procédés de conservation et de détoxication ont conduit les scientifiques à chercher des fongicides naturels, sans risque et respectueux de l'environnement. Dans ces recherches menées en Afrique, l'extrait de la feuille de Lippia multiflora a montré un effet statique fongique sur Aspergillus flavus et Fusarium verticillioides (Anjorin et al., 2008). Les huiles essentielles, l'ozone, la terre à diatomées et les antioxydants alimentaires tels que l'hydroxyanisole butylé (BHA), l'hydroxytoluène butylé (BHT) et le parabène de propyle (PP) sont des options rentables non toxiques et prometteuses qui peuvent remplacer les conservateurs chimiques toxiques dans la lutte contre divers champignons y compris Aspergillus, Fusarium et Penicillium (Chulze, 2010). Au Bénin par exemples une revue des écrits scientifiques publiés faite par Ba et al. (2015) a révélé une large gamme de produits chimiques utilisés pour inhiber la production d'aflatoxine dans les stocks de maïs en y limitant la prolifération des moisissures. Parmi ces produits nous pouvons noter l'acide propionique $(0,1-0,5 \%)$, l'ammoniac $(0,5 \%)$, le copper sulphate $(0,5-1 \%)$ et l'acide benzoïque $(0,1-0,5 \%)$ qui inhibent complètement la croissance de $A$. parasiticus ; le benzoate de sodium quant à lui, a un effet antimicrobien sur la croissance de Aspergillus niger, Aspergillus flavus et Aspergillus fumigatus. L'hypochlorite de sodium $(0,1-$ $0,5 \%)$ expose une propriété antifongique (68$4 \%$ ). Il a été démontré dans cette revue que les extraits de plante comme les composants du neem (Azadirachta indica) et la scopolétine qui est extraite à partir de plusieurs plantes principalement dans Nicotiana glauca, Lycium chinense, Angelica dahurica et les racines de Trigonella foenum-graecum, ont des propriétés antifongiques et inhibent aussi la production d'aflatoxine. Par rapport au riz non traité, l'utilisation de certains extraits de plantes a pu réduire le niveau de l'aflatoxine B1 de 99\% (Reddy et al., 2009).

Les huiles essentielles peuvent être aussi appliquées sous forme de vapeur, ce qui rend l'application particulièrement pratique et convenable pour une utilisation dans des entrepôts fermés (Chulze, 2010). 


\section{Les méthodes biologiques}

L'une des stratégies les plus prometteuses à long terme en matière de lutte contre les aflatoxines est le développement de variétés résistantes. Des lignées de maïs résistantes aux aflatoxines ont été identifiées et introduites dans les programmes publics et privés de sélection végétale (Wagacha et Muthomi, 2008). Dans le cadre d'une stratégie de collaboration Etats-Unis-Afrique, l'Institut international d'agriculture tropicale (IITA) et l'USDA ont obtenu six lignées pures adaptées à l'Afrique et présentant une meilleure résistance à l'accumulation d'aflatoxines (Menkir et al., 2006 ; 2008).

En Afrique, des expériences de lutte biologique contre les aflatoxines utilisant aussi des souches locales sont en cours au Kenya (Okun et al., 2015; Chebon et al., 2016) et au Nigeria, mais à un stade plus avancé dans ce dernier pays, où les cultivateurs de maïs sont parvenus à réduire d'environ $80 \%$ la contamination par les aflatoxines (IITA, 2010). Dans une étude menée au Nigéria, l'inoculation pendant deux années de suite d'un mélange de quatre souches atoxinogénes endémiques d'A. flavus dans des parcelles de maïs situées dans quatre contextes agro-écologiques différents a entraîné une réduction significative des concentrations d'aflatoxine au moment de la récolte et après stockage (Atehnkeng et al., 2014). Au moment de la récolte et après stockage, la réduction de la teneur en aflatoxine est passée de 57,2\% (27,1 ppb dans les parcelles non traitées contre 11,6 dans les parcelles traitées) à 99,2\% (2792,4 ppb dans les parcelles non traitées contre 23,4 ppb dans les parcelles traitées).

Les souches atoxinogénes sont restées dans les maïs traités, et les concentrations d'aflatoxines dans les grains après stockage dans de mauvaises conditions, ont diminué de 93,5\% (956,1 ppb dans le maïs non traité contre $66,2 \mathrm{ppb}$ dans le maïs traité) à $95,6 \%$ (2408,3 ppb dans le maïs non traité contre 104,7 dans le maïs traité).

Certaines techniques de prise en charge post-récolte des céréales ont permis d'y réduire la concentration d'aflatoxine.
Kaaya et Kyamuhangire (2010), ont montré lors de leur étude sur l'effet des séchoirs à convecteurs naturels chauffés par la biomasse dans l'amélioration de la qualité du maïs en Ouganda, que ces séchoirs réduisait sa contamination par les moisissures et par les aflatoxines, et n'altérait pas la capacité de germination des graines. Ce mode de séchage s'est avéré également très efficace contre les pertes dues aux dégâts des insectes.

Dans le cas du maïs en Afrique, le tri manuel est modérément efficace au niveau des villages pour sélectionner les lots de grains dont la teneur en aflatoxine est plus faible. L'élimination manuelle des grains visiblement moisis, abîmés par les insectes et cassés a permis de réduire de $40 \%$ la teneur en aflatoxine, d'après une étude effectuée au Bénin (Fandohan et al., 2005). Le risque de contamination des aliments de consommation humaine et animale par les aflatoxines en Afrique est accentué par les facteurs environnementaux, agronomiques et socioéconomiques (Hell et Mutegi, 2011). Les pratiques de gestion agricole qui augmentent les rendements des cultures appliquées en Afrique réduisent le risque de formation d'aflatoxine. Ces pratiques comprennent l'utilisation de variétés résistantes aux insectes comme Cathartus quadricollis et Sitophilus zeamais qui jouent un rôle important dans la contamination des aliments par les moisissures toxinogénes selon Hell et al. (2003); Lamboni et Hell (2009), la rotation des cultures, la plantation au moment propice, la lutte contre les plantes et animaux nuisibles, l'irrigation et la fertilisation pour palier la sécheresse et le stress nutritionnel.

Depuis quelques années une nouvelle technologie de contrôle au champ de l'aflatoxine est utilisée au Nigéria, au Kenya, au Sénégal et au Burkina Faso. Cette technologie dénommée AflaSafe est basée sur le principe d'exclusion compétitive qui atténue les effets négatifs de l'aflatoxine. Son utilisation a donné des résultats probants sur le maïs au Kenya (Marechera et Ndwiga, 2012).

\section{Les méthodes physiques}


Les méthodes physiques sont nombreuses. Elles sont basées en général sur le lavage, le séchage, le broyage, le tri manuel, la séparation mécanique ou le traitement thermique

Les mycotoxines sont en général thermostables et elles résistent à tous les procédés utilisés pour l'élimination des microorganismes (chauffage et stérilisation). Peers et Linsell (1975) observaient que les aflatoxines restent stables dans les arachides ou dans le maïs après un chauffage à $200{ }^{\circ} \mathrm{C}$ pendant 30 minutes. Il est à noter que les traitements thermiques dépendent en grande partie d'autres facteurs tels la teneur de l'aliment contaminé en eau et de son $\mathrm{pH}$ (Rustom, 1997). L'irradiation a été considérée longtemps comme une solution possible de lutte contre les microorganismes. Des méthodes satisfaisantes pour l'élimination des mycotoxines ne sont pas encore mises au point. Cependant l'irradiation peut être envisagée pour lutter contre les moisissures toxinogènes.

\section{LES LIMITES DE LA LUTTE CONTRE L'AFLATOXINE EN AFRIQUE}

Il est avéré que les études expérimentales des différentes méthodes de lutte contre la présence d'aflatoxine dans les céréales ont donné des résultats probants. Mais en Afrique beaucoup de contraintes freinent l'application de ces méthodes. En effet, la filière céréalière est généralement l'apanage de petits agriculteurs en Afrique. Ces petits exploitants ne disposent généralement pas d'informations précises sur les facteurs favorisant la prolifération des moisissures aflatoxinogènes ni sur les effets de la toxine sur la santé humaine et animale (Kang'ethe et Lang'at, 2009). La sensibilisation des cultivateurs sur les risques sanitaires associés à l'aflatoxine et sur les moyens de réduire l'exposition dépend de leur situation socioéconomique, de leur éducation, de la taille de leur exploitation, de leur participation à la vulgarisation agricole, de l'orientation du marché, de la motivation économique et de leurs perceptions (Adegoke et Letuma, 2013). Les résultats d'une enquête menée dans les zones rurales d'Afrique du Sud au cours de laquelle Mboya et Kolanisi (2014) ont interviewé 260 familles de petits cultivateurs, montrent que peu de fermiers sont conscients des risques sanitaires liés aux mycotoxines. Des résultats similaires ont été obtenus dans une étude beaucoup plus importante (taille de l'échantillon : 2400) réalisée au Bénin, au Ghana et au Togo (James, 2005). Ces résultats démontrent que les pratiques agricoles en Afrique visent l'autosuffisance alimentaire sans se préoccuper de la présence d'aflatoxine dans les aliments. Par exemple en Afrique subsaharienne, $80 \%$ des exploitants agricoles sont de petits cultivateurs, pratiquant une agriculture de subsistance (Mboya et Kolanisi, 2014).

La principale préoccupation de ces exploitants est la sécurité alimentaire. En Afrique, la sécurité alimentaire représente la principale barrière à la mise en place de certaines méthodes de lutte contre l'aflatoxine telle que le tri (Fandohan et al., 2008).

En plus de cette problématique de l'éducation en matière de sécurité sanitaire des aliments des exploitants céréaliers, la lutte contre l'aflatoxine dans les céréales manque de volonté politique dans la quasi-totalité des pays Africain. En Afrique l'aflatoxine n'est pas une priorité politique. De ce fait les programmes de lutte contre ce fléau ne sont pas intégrés dans les chaines de valeur céréalière. La prévention et le contrôle de l'aflatoxine s'ils existent en Afrique ne concernent que la production céréalière destinée à l'exportation.

Aussi, l'Afrique est confrontée à un manque d'experts capables de mener à bien certaines méthodes de lutte contre l'aflatoxine comme l'utilisation des espèces céréalières génétiquement résistant à l'aflatoxine ou encore l'emploi des espèces atoxinogènes d'aspergillus. Ces méthodes sont aléatoires et soumises aux effets de l'environnement. En plus des ces différentes contraintes, le coût financier de la lutte contre l'aflatoxine en particulier la lutte biologique est élevé. 


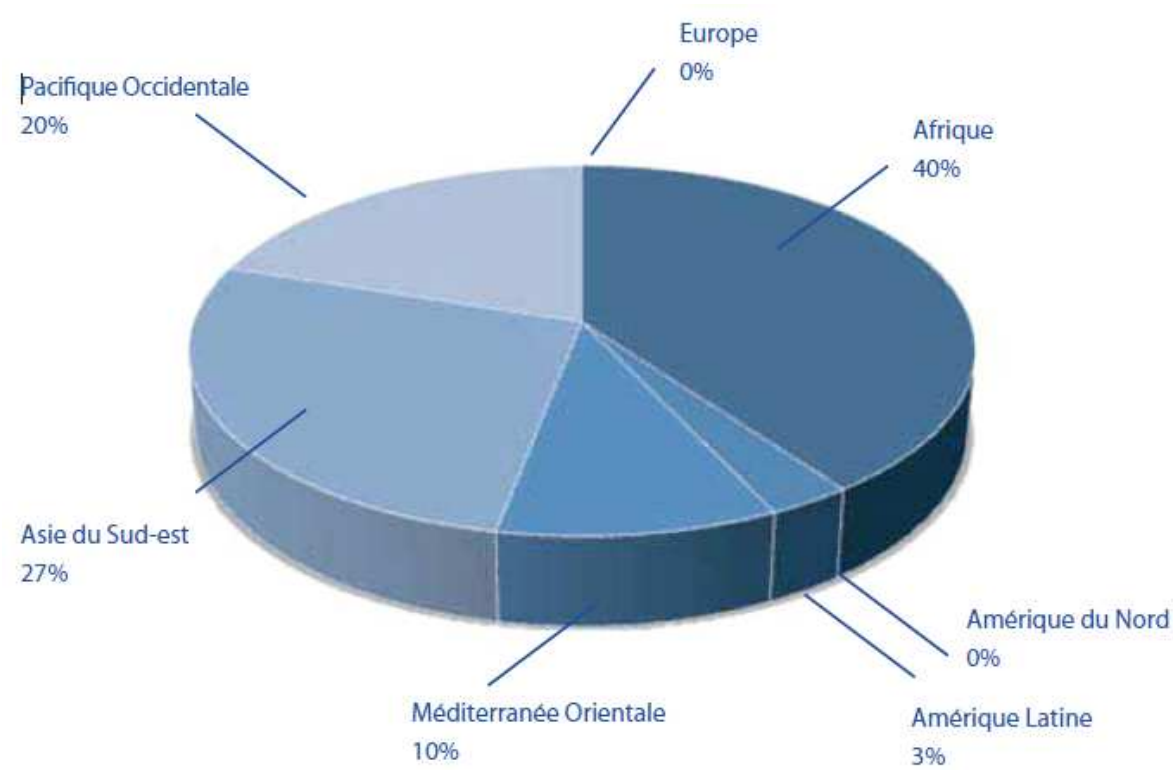

Figure 1: Répartition des Hépato-carcinomes attribuables à l'aflatoxine dans le monde. Source : Yu et Liu (2010).

Tableau 1: Retard de croissance des enfants dans quelque pays d'Afrique corrélé à leur exposition à l'aflatoxine.

\begin{tabular}{lcc}
\hline Pays & $\begin{array}{c}\text { Exposition à l'aflatoxine } \\
\text { ng/kg de masse corporelle/jour }\end{array}$ & $\begin{array}{c}\text { Pourcentage de retard de } \\
\text { croissance chez les Enfants }\end{array}$ \\
\hline Gambie & $4-115$ & 28 \\
Kenya & $3.5-133$ & 36 \\
Nigéria & $139-227$ & 43 \\
Tanzanie & $0,02-50$ & 44 \\
\hline \multicolumn{1}{c}{ Source : Khlangwiset et al. (2011) } &
\end{tabular}

\section{Conclusion}

Nous observons que la prévalence de la contamination des céréales est très élevée en Afrique.

Les conditions climatiques des régions touchées, le stress subi par la plante, les mauvaises pratiques culturales et les dommages causés par les insectes et les ravageurs favorisent l'infection par Aspergillus et la production d'aflatoxines dans ces denrées de première nécessité. L'ampleur et les conséquences de la contamination par l'aflatoxine font des ravages dans le continent sur le plan économique, et sanitaire et sont toujours d'actualités.

Ainsi des recherches approfondies dans l'optique de réduire significativement voir éradiquer la contamination des céréales par l'aflatoxine méritent d'être menées dans tous les pays africains. Mais le préalable à toute forme de lutte contre la contamination des céréales par l'aflatoxine est la sensibilisation des petits producteurs. Les pouvoirs publics devront montrer une réelle volonté de lutter contre ce fléau, qui reste un problème de santé publique, en allouant un budget plus conséquent pour la recherche et sensibiliser les producteurs sur l'existence des mycotoxines surtout l'aflatoxine en insistant sur leur effets néfastes sur la santé humaine et animale.

\section{CONFLIT D'INTERETS}

Les auteurs déclarent qu'ils n'ont aucun conflit d'intérêts. 


\section{CONTRIBUTION DES AUTEURS}

Ce travail est fait dans le cadre de la thèse de doctorat de ED encadré par DT, IS et MS avec la participation de DRF qui étudie les conditions de production d'aflatoxine dans le Sorgho et de FS responsable de l'atelier céréales et légumineuses de l'ITA.

\section{REMERCIEMENTS}

Tous les auteurs remercient l'Institut de Technologie Alimentaire du Sénégal (ITA), structure au sein de laquelle nous effectuons nos travaux de recherche. Nos remerciements vont aussi au Dr Amadou KANE qui a bien voulu lire et corriger notre document, mais également au Dr Djibril TRAORE et ces collaborateurs (Ibrahima SARR et Charles P.WOLOSHUK) qui ont eu l'amabilité de prendre en charge l'aspect financier de nos travaux dans le cadre des projets FPL (Food Processing Lab) et SMIL (Sorghum and Millet Innovation Laboratory).

\section{REFERENCES}

Abebe Menkir R, Brown L, Bandyopadhyay R, Cleveland TE. 2008. Registration of Six Tropical Maize Germplasm Lines with Resistance to Aflatoxin Contamination. J. of Plant Reg., 2(3): 246-50.

Abu Agla S. 2002. Seed borne fungi of important food crops of the Gezira Scheme, Sudan. MSc. Thesis, University of Khartoum, Sudan.

Adhikari M, Gita-Ramjee, Berjak P. 1994. Aflatoxin, kwashiorkor, and morbidity. Natural Toxins, 2(1):13.

Adegoke GO, Letuma P. 2013. Strategies for the Prevention and Reduction of Mycotoxins in Developing Countries. In Mycotoxin and Food Safety in Developing Countries, Makun HA (Ed). InTech, Rijeka : Croatia ; 123-136.

AfricaAIMS(Africa Aflatoxin Information Management System), C-SAAP (Soutien du Plan D'action et d'Analyse des Aflatoxine). 2016. Results Updates on AfricaAIMS and C-SAAP.
Afssa. 2009. Évaluation des risques liés à la présence de mycotoxines dans les chaînes alimentaires humaine et animale. Rapport final.

Ahmed NE, Abu Agla, Idris M, Elhussein S. 2005. Fungal contamination of Sorghum grains, a possible threat to grain quality. Proceedings of 1 st work shop in mycotoxins related health disorders in Sudan, 18-21 April, Khartoum, Sudan, p 1-12. Sudanese Standards and Metrology Organization in collaboration with Wageningen University, The Netherlands.

Ahmed NE, Abu Agla S, Idris MO, Elhussein S. 2008. Fungi associated with stored Sorghum grains and their effects on grain quality. Life Sci., 2(3): 723-729.

Ahmed, NE, Abdalla AE, Adam YS, Betjowck. 2009. Fungi and mycotoxins associated with Sorghum grains in major storage systems in Gedarif, Sudan. A paper submitted to the 17th Board of Directors meeting of the national council for mycotoxins, December 2009, Sudanese Standards and Measurements Organisation, Sudan.

Alakonya AE, Monda EO, Ajanga S. 2009. Fumonisin B1 and Aflatoxin B1 levels in Kenya maize. J. Plant Pathol., 91(2): 459-464.

AnjorinST, Makun, HA, Iheneacho HE. 2008. Effect of Lippia Multiflora leaf extract and Aspergillus flavus on germination and vigour indices of Sorghum Bicolor [L] (Moench). Int. J. Trop. Agric. Food Syst., 2(1): 130 - 134 .

Atehnkeng J, Ojiambo PS, Cotty PJ, Bandyopadhyay R. 2014. Field efficacy of a mixture of atoxigenic Aspergillus flavus vegetative compatibility groups in preventing aflatoxin contamination in maize (Zea mays L.). Biol. Contr., 72: 62-70.

Atehnkeng J, Ojiambo PS, Donner M, Ikotun K, Sikora RA, Cotty PJ, Bandypadhyay R. 2008. Distribution and toxicity of Aspergillus species isolated from maize kernels from three agro-ecological zones 
of Nigeria. Int. J. Food Micr., 122: 7484.

Azziz-Baumgartner E, Lindblade K, Gieseker $\mathrm{K}$, Rogers HS, Kieszak S, Njapau H, Schleicher R, McCoy LF, Misore A, DeCock K, Rubin C, Slutsker L, Aflatoxin Investigative Group. 2005. Case-Control Study of an Acute Aflatoxicosis Outbreak, Kenya. Environ. Health Perspect., 113(12): 1779-1783.

Ba R, Monteiro NMF, Koudjega H, Adjagbo C, kohoude J, Djinadou Igue F, Gbaguidi F, Mensah GA, Baba Moussa L. 2015. Synthèse bibliographique sur l'utilisation de la scopolétine pour la réduction des aflatoxines du maïs en stock au bénin. Ann. Sci. Agronom., 19(2): 201-211.

Bandyopadhyay R, Kumar M, Leslie, JF. 2007. Relative severity of aflatoxin contamination of cereal crops in West Africa. Food Additives and Contaminants, 24(10): 1109-1114.

Bankole MO, Omemu AM, Adegbesan AM. 2006. Maize cob as a microbiological growth medium for fungi. ASSET, Series B, 5(1): 57- 64 .

Bankole SA, Adebanjo A. 2003. Mycotoxins in food in West Africa: current situation and possibilities of controlling it. Afr. J. Biotechn., 2(9): 254-263.

Bryden WL. 2007: Mycotoxins in the food chain: human health implications. Asia Pacific J. Clinical Nutr., 16(1): 95-101.

CDC (Center for Disease Control). 2004. Epidémie d'intoxication par les aflatoxines - provinces orientale et centrale, du Kenya, Janvier - Juillet 2004. Morbidity and Mortality Weekly Report, 53(34): 790-793.

Chebon S, Wanyoike W, Bii C, Gathumbi J, Ogoyi D. 2016. Incidence of Aflatoxigenic Fungi and Aflatoxins in Maize Cultivated in Highland and Midaltitude Agro-ecological Zones in Kenya. J. Scientific Res. Reports, 9(6): 1-12.

Chulze SN. 2010. Les stratégies visant à réduire les niveaux de mycotoxines dans le maïs pendant le stockage: A review.
Food Additives and Contaminants, 27(5): 651- 657 .

CODEX. 2013. CODEX 193-1995. CODEX general standards for contaminants and toxins in food and feed. http://www.codexalimentarius.org/input/d ownload/standards/17/CXS 193e.pdf. Consulté en Juin 2016.

DN (Daily Nation) newspaper, October $4^{\text {th }}$. 2001. Publication of Nation Media, Nairobi, Kenya.

ECOWAS. 2015. Etude exploratoire pour l'évaluation de l'environnement politique et des capacités techniques pour le contrôle des aflatoxines dans les états membres de la CEDEAO.

Elegbede JA. 1978. Fungal and mycotoxin contamination of Sorghum during storage.M.sc thesis submitted to department of Biochemistry, Ahmadu Bello University, Zaria.

Fandohan P, Hell K, Marasas WF. 2008. Food processing to reduce mycotoxins in Africa. Pre- and postharvest management of aflatoxin in maize. In Mycotoxins: Detection Methods, Management, Public Health and Agricultural Trade, Leslie JF, Bandyopadhyay R, Visconti A (Eds). CABI Publishing: Wallingford, UK; 302309.

Fandohan P, Zoumenou D, Hounhouigan DJ, Marasas WF, Wingfield MJ, Hell K. 2005. Fate of aflatoxins and fumonisins during the processing of maize into food products in Benin. Int. J. Food Microbiol., 98: 249-259.

FAOSTAT. 2015. "Food and Agricultural Organisation of the United Nation". FAOSTAT Database available on line http://faostat fao.org/site/567/default.aspx (20.08.2015)

Fellinger A. 2006. Worldwide mycotoxin regulations and analytical challenges. World Grain Summit: Foods and Beverages, San Francisco, California, USA.

Gong YY, Cardwel LK, Hounsa A, Egal S, turner PC, Hall A J, Wild, C.P. 2002. Exposition à l'aflatoxine alimentaire et la 
croissance avec facultés affaiblies chez les enfants du Bénin et du Togo: étude transversale. BMJ, 325(7354): 20-21.

Gong YY, Egal S, Hounsa A, Turner PC, Hall AJ, Cardwell KF. 2003. Determinants of aflatoxin exposure in young children from Benin and Togo, West Africa: the critical role of weaning. Int. J. Epidemiol., 32: 556-562.

Gong Y, Hounsa A, Egal S. 2004. Postweaning exposure to aflatoxin results in impaired child growth: a longitudinal study in Benin, West Africa. Environmental Health Perspective 112: 1334-38.

Hell K, Mutegi C. 2011. Aflatoxin control and prevention strategies in key crops of Sub-Saharan Africa. Afric. J. Microbiol. Res., 5(5): 459-66.

Hell K, Cardwell K, Poehling H. 2003. Relationship between management practices, fungal infection and aflatoxin for stored maize in Benin. J. Phytopath., 151: 690-698.

Huffman J, Gerber R, Du L. 2010. "Recent advancements in the biosynthetic mechanisms for polyketide-derived mycotoxins. Biopolymers 93(9): 764-76.

IARC (International Agency for Research on Cancer). 1997. Polychlorinated dibenzopara-dioxins and polychlorinated dibenzofurans. IARC Monogr Eval Carcinog Risks Hum, 69: 1-631.

IARC. 2012. Chemical agents and related occupations. IARC Monogr Eval Carcinog Risks Hum, 100: 9-562.

ICRISAT(Institut international de recherche sur les cultures des zones tropicales semiarides ). 2002. Aflatoxin contamination of groundnut - health and economic implications for India's rural poor. International Crops Research Institute for the Semi-Arid Tropics (www.icrisat.org International Rice Research Institute (IRRI, 1995). World Rice Statistics (1993-94).

IITA (International Institute of Tropical Agriculture). 2010. Making Kenyan maize safe from deadly aflatoxins. 05
June 2010., Ibadan, Nigeria. http://old.iita.org/cms/details/news_detail s.aspx.

James B. 2005. Conscientisation du public sur l'aflatoxine et Controle de qualité des aliments. Benin: international institute of tropical Agriculture.

Javis B. 1971. Factors affecting the production of mycotoxins. J. Appl. Bact. 34(1): 199-213.

Kaaya AN, Kyamuhangire W. 2010. Drying maize using biomass-heated natural convection dryer improves grain quality during storage. J. App. Sci., 11: 967-974.

Kane A, Ndir B, Sarr AB, DIOP N, Mané Y, Diack TS. 1991. Occurrence de l'aflatoxine B1 dans les principales denrées alimentaires vendues sur les marchés sénégalais. In Alimentation et Nutrition dans les Pays en Développement (4ème journées internationales du GERM), Lemonnier D, Ingenbleek $\mathrm{Y}$ et Hennard $\mathrm{Ph}$ (eds) KARTHALA-ACCT-AUPELF: Paris; 143-148.

Kang'ethe, EK, Lang'at AK. 2009. Aflatoxin $\mathrm{B} 1$ and M1 contamination of animal feeds and milk from urban centers in Kenya. Afric. Health Sci., 9(4): 218-226.

Khlangwiset P, Shephard GS, Wu F. 2011. Aflatoxins and growth impairment: a review. Crit. Rev. Toxicol., 41(9): 74055.

Kimanya ME, De Meulenaer B, Tiisekwa B, Ndomondo Sigonda M, Devlieghere F, Van Camp J. 2008. Co-occurrence of fumonisins with aflatoxins in homestored maize for human consumption in rural villages of Tanzania. Food Addit Contam Part A. Chem. Anal. Control. Expo. Risk Assess, 25: 1353-1364.

Lamboni Y, Hell K. 2009. Propagation of mycotoxigenic fungi in maize stores by post-harvest insects. Int. J. Food Microbiol., 29: 31-39.

Lee LS, Dunn JJ, De LuccaA J, Ciegler A. 1981. Role of lactone ring of aflatoxin B1 in toxicity and mutagenicity. Experientia, 37: 16-17. 
Lewis L, Onsongo M, Njapau H, SchurzRogers H, Luber G, Kieszak S. 2005. Aflatoxin contamination of commercial maize products during an outbreak of acute aflatoxicosis in eastern and central Kenya. Environ. Health Perspect., 113(12): 1763-1767.

Liu Y, Wu F. 2010. La Charge Mondiale du carcinoma hépatocellulaire carcinome induite par l'Aflatoxine: Une évaluation des risques. Environ. Health Perspect., 118(6): 818-824.

Makun HA, Dutton MF, Njobeh PB, Mwanza M, Kabiru AY. 2011. Natural multimycotoxin occurrence in rice from Niger State. Nigeria Mycotoxin Res., 27(2): 97104.

Makun HA, Gbodi TA, Akanya HO, Sakalo AE, Ogbadu HG. 2007. Fungi and some mycotoxins contaminating rice (Oryza sativa) in Niger state, Nigeria. Afric. J. Biotechnol., 6(2): 99-108.

Makun HA, Gbodi TA, Akanya HO, Salako EA, Ogbadu GH. 2009. Fungi and some mycotoxins found in mouldy Sorghum in Niger State, Nigeria. World J. Agri. Sc., 5(1): 05-17.

Marechera G, Ndwiga J. 2012. Estimation of the potential adoption of Aflasafe among smallholder maize farmers in lower eastern Kenya. Afric. J. Agric. Resource Econ., 10(1): 72-85.

Mboya RM, Kolanisi U. 2014. Subsistence Farmers' Mycotoxin Contamination Awareness in the SADC Region: Implications on Millennium Development Goal 1, 4 and 6. J. Human Ecol., 46(1): 21-31.

Menkir A, Brown RL, Bandyopadhyay R, Chen ZY, Cleveland TE. 2006. A USAAfrica collaborative strategy for identifying, characterizing, and developing maize germplasm with resistance to aflatoxin contamination. Mycopathologia, 162(3): 225-32.

Moss MO. 2008. Les questions liées aux champignons, à la qualité et à la sécurité dans les fruits et légumes frais. J. App. Microbiol., 104 (5) 1239-1243.
Murphy PA, Hendrich S, Landgren C, Bryant CM. 2006. Food mycotoxins: An update. J. Food Sci., 71: 51-65.

Muthama J, Muthomi W, Njenga LN, Gathumbi JK, Chemining'wa GN. 2009. The occurrence of aflatoxins in maize and distribution of mycotoxin-producing fungi in Eastern Kenya. Plant Pathol. J., 8(3): 113-119.

Muture BN, Ogana G. 2005. Aflatoxin levels in maize and maize products during the 2004 food poisoning outbreak in Eastern Province of Kenya. East Afr. Med. J., 82: 275-279.

Njobeh BP, Dutton FM, Makun HA. 2010. Mycotoxins and human health: Significance, prevention and control In Smart Biomolecules in Medicine, Ajay KM, Ashutosh T, Shivani BM (Eds). VBRI Press: India; 132-177.

Obade MI, Andang'o P, Obonyo C, Lusweti F. 2015. Exposure of children 4 to 6 months of age to aflatoxin in kisumu country, Kenya. Ajfand, 15(2).

Okun DO, Khamis FM, Muluvi GM, Ngeranwa1 JJ, Ombura FO, Yongo MO, Eucharia U. 2015. Distribution of indigenous strains of atoxigenic and toxigenic Aspergillus flavus and Aspergillus parasiticus in maize and peanuts agro-ecological zones of Kenya. Agric. Food Secur., 4: 14. DOI 10.1186/s40066-015-0033-5.

Omondi Odhiambo B, Hunja Murage, Nyokabi Wagara I. 2013. Isolation and characterisation of aflatoxigenic Aspergillus species from maize and soil samples from selected counties of Kenya. Afric. J. Micro. Res., 7(34): 4379-4388.

Oppong Akowuah1 J, Dzifa Mensah1 L, Chian Chan, Roskilly A. 2015. Effects of practices of maize farmers and traders in Ghana on contamination of maize by aflatoxins: Case study of EjuraSekyeredumase Municipality. Afric. J. Microbio., 9(25): 1658-1666.

Peers FG, Linsell CA. 1975. Aflatoxin contamination and its heat stability in Indian cooking oil. Trop. Sci., 17: 229. 
Probst C, Njapau H, Cotty PJ. 2007. Outbreak of an acute aflatoxicosis in Kenya in 2004: identification of the causal agent. Appl. Environ. Microbiol., 73(8): 27622764.

Reddy KRN, Reddy CS, Muralidharan K. 2009. Potentiel des agents botaniques et de lutte biologique sur la croissance et la production d'aflatoxine par Aspergillus flavus infectant les grains de riz. Food Control, 20: 173-178.

Rustom IYS. 1997. Aflatoxin in food and feed: Occurrence, legislation and inactivation by physical methods. Food Chem., 59: 57-67.

Ryle M. 2010. Ergot affected and mouldy sorghum.Department of employment, economic development and innovation,Australia. Primary industries and fisheries 28 .

Shephard GS. 2003. Aflatoxin and food safety: recent African perspectives. Toxin Rev., 22(2-3): 267-286.

Shephard GS. 2008. L'évaluation des risques des aflatoxines dans les aliments en Afrique. Les additifs alimentaires et les contaminants: Partie A: Chimie, analyses, contrôle, Évaluation de l'exposition et des risques. 25(10): 1246-1256.

Strosnider H, Azziz-Baumgartner E, Banziger $\mathrm{M}$, Bhat RV, Breiman R, Brune $\mathrm{M}$, Decock K, Dilley A, Groopman J, Hell K, Henry SH, Jeffers D, Jolly C, Jolly P, Kibata GN, Lewis, Liu X, Luber G, Mccoy L, Mensah P, Miraglia M, Misore A, Njapau H, Ong C, Onsongo, MTK. Page SW, Park D, Patel M, Phillips T, Pineiro M, Pronczuk J, Schurz Rogers H, Rubin C, Sabino M, Schaafsma A,
Shephard G, Stroka J, Wild C, Williams JT, Wilson D. 2006. Rapport du groupe de travail: Les stratégies de Santé Publique visant à réduire l'exposition aux aflatoxines dans les pays en voie de développement. Environ. Health Perspect., 114: 1989-1903.

Tabuc C. 2007. Flore Fongique de Différents Substrats et Conditions Optimales De Production Des Mycotoxines. Thèse De Doctorat D'université : Pathologie, Mycologie, Génétique Et Nutrition. Toulouse : L'institut National Poly Technique Et De L'université De Bucarest, France, 190p.

VargaJ, Frisvad JC, Samson RA, 2011. Two new aflatoxin producing species, and an overview of Aspergillus section Flavi. Stud. Mycol. 69: 57-80.

Wagacha JM. JW Muthomi, 2008. Mycotoxin problem in Africa current status implications to food safety and health and possible management strategies. Int. J. Food Microbiol., 124: 1-12.

Watson S, Diedhiou PM, Atehnkeng J, Dem A, Bandyopadhyay R, Srey C, Routledge MN, Gong YY. 2015. Seasonal and geographical differences in aflatoxin exposures in Senegal. World Mycotoxin J., 8(4): 525-531.

Wild CP, Gong YY. 2010. Mycotoxins and human disease: a largely ignored global health issue. Carcenogensis, 31: 71-82.

Williams JH, Phillips TD, Jolly PE, Stiles JK, Jolly CM, Aggarwal D. 2004. Human aflatoxicosis in developing countries: a review of toxicology, exposure, potential health consequences, and interventions. Am. J. Clin. Nutr., 80: 1106-1122. 